\title{
Optimal design of cutting-disc of no-tillage planter based on kinematics analysis and FEA
}

\author{
ZHAO Xu , CHEN Dong \\ School of Mechanical Engineering and Automation, University of Science and Technology Liaoning, China
}

\begin{abstract}
Excellent performances of cutting and anti-blockageis required for the no-tillage planter, a new model of cutting-disc with oblique ripples has been developed. Obliquity angle of ripple has a strong impact onworking performance, so kinematics analysis of discs with different ripple obliquity angle is conducted through ProE, the result shows that $30^{\circ}$ is the optimum angle. And FEA shows that the largest stress is on the ripple which splits the soil, reasonable thermal treatment plan is drawn up based on the FEA.
\end{abstract}

\section{Introduction}

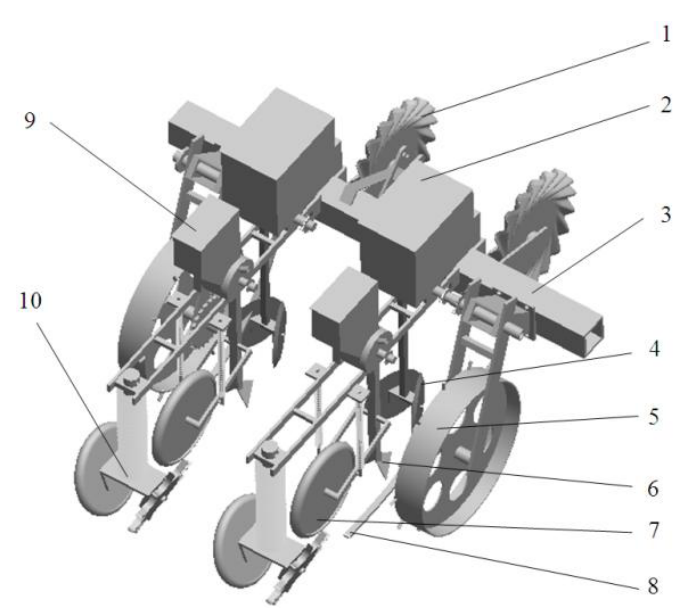

1. cutting-residues disc 2 . fertilizer feeder3. Rack

4. stagger double-disk styled opener5. land wheel

6. Sharp-angled opener 7.suppress wheal

8. anti-blocking flat spring 9. hacking and covering shovel 10.hacking and covering shovel

Figure 1. Assembly drawing of No-tillage planter

Conservation tillage technology plays an integral role in increasing moisture in the soil for seeding, one of key equipment for no-tillage mechanization is no-tillage planter with extraordinary ability of cutting-residues and anti-blocking2,3. As shown in Figure.1, no-tillage planter consists of rack 3, cutting-residues disc 1, fertilizer feeder 2, stagger double-disk styled opener 4 , hacking and covering shovel 9, Sharp-angled opener 6, anti-blocking flat spring 8, land wheel 5,suppress wheal 7 and hacking and covering shovel 10.

Aiming anti-blocking,a type of cutting-disc with oblique ripples, having the excellent capacity of cutting beneath surface soil and stubble, is introduced, it not only provides seed ditch of sufficient width and depth, but also ensures minimum disturbance soil, and it would not throws soil, seeing the working process in Figure.2. The incidence

angleofrippleshave astrong impactonworking performanc e, so kinematics of cutting-discs, with different incidence angles, was analyzedbased on ProE, to obtain optimum incidence angle. And Finite element stress analysis of ANSYS presents the disc stress distribution diagram, offering solutions for thermal treatment plan and surfacestrengthening.

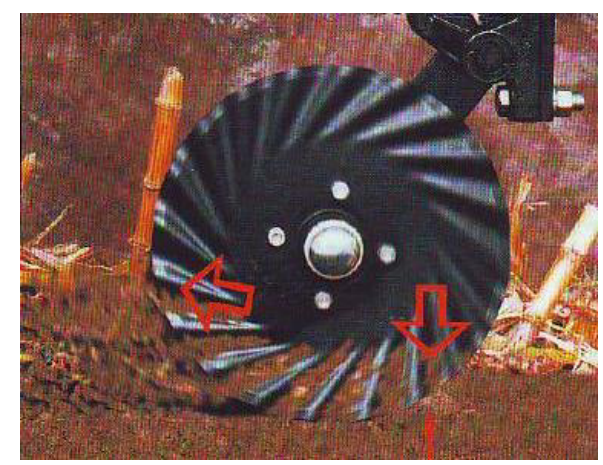

Figure 2.Working Process of the cutting-disc

\section{Kinematics analysis of cutting-disc}

\subsection{Elementary structure size of cutting disc}

According to the agronomic requirements, good cutting effect for corn stubble requires cutting speed of above $2 \mathrm{~m} / \mathrm{s}$, so $V=2.5 \mathrm{~m} / \mathrm{s}$ is selected as the linear velocity of 
disc border. Physical properties of maize root crop in northeast China determines cutting-depth $h=100 \mathrm{~mm}$.In terms of Structural integrity requirements and field traffic ability, disc radius, $\mathrm{R}=220 \mathrm{~mm}$, is selected. To ensures strong cutting ability, It isdesired that ripple cuts soil and stubble verticallyat initial contacting,and the ripple length should be longer than the cutting-depth, hence, $r=130 \mathrm{~mm}$ is selected as inner radius of ripples. Tests showed that $L=55 \sim 70 \mathrm{~mm}$ is appropriate for groove clearance, based on $\mathrm{L}=2 \pi R / \mathrm{z}$, so number of ripples is $z=40$. Thickness of disc is set as $\delta=5 \mathrm{~mm}$ by reference to the criterion NJ163-78. Research by Great Plains (Great Plains Manufacturing, 2000) shows that appropriate value for width amplitude is $A=8 \sim 15 \mathrm{~mm}$, so $\mathrm{A}=10 \mathrm{~mm}$ is selected based on an informed choice.

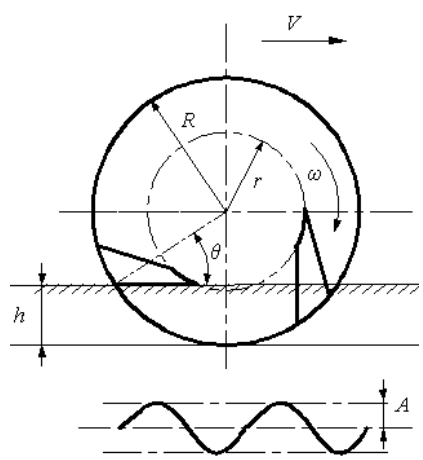

Figure 3.The structure parameter diagram of cutting disc

The feasible incidence angle of ripples is $\theta=0^{\circ}, \theta=30^{\circ}$, $\theta=45^{\circ}$, according to experiment and agronomic requirements. Kinematics analysis of the three types of cutting-disc is conducted in another section below, to obtain optimum angle of ripples.

\subsection{D modelling and location of measurement point}

Three types of 3D model of cutting-discs, respectivelywithincidenceangle of ripples $\theta=0^{\circ}, \theta=30^{\circ}$ and $\theta=45^{\circ}$, is built by ProE. For all types of $3 \mathrm{D}$ model, marking pattern of measurement point is shown in Fig.4. Four equidistant points, respectively labeled 1,2,3,4, were marked on a ripple to analysis the cutting motions of different points on a given ripple, the same analytic process is conducted for the three types of cutting-discs to determine the optimized incidence angle.

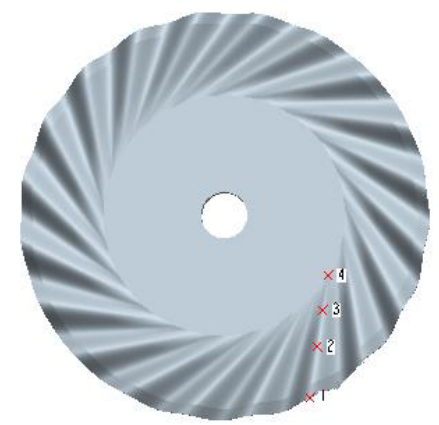

Figure 4.Modelling by Pro/E and location of measurement points

\subsection{Movement simulation}

The choice of orientation of the $(\mathrm{x}, \mathrm{y}, \mathrm{z}) \quad$ Cartesian coordinatesystem is set as follow:working direction of no-tillage planter is in the direction of the positive axis $\mathrm{x}$, upward direction perpendicular to the ground, along the radial direction, is the direction of the positive axis $y$, and the direction of the positive axis $\mathrm{z}$ is set by left-hand rule.

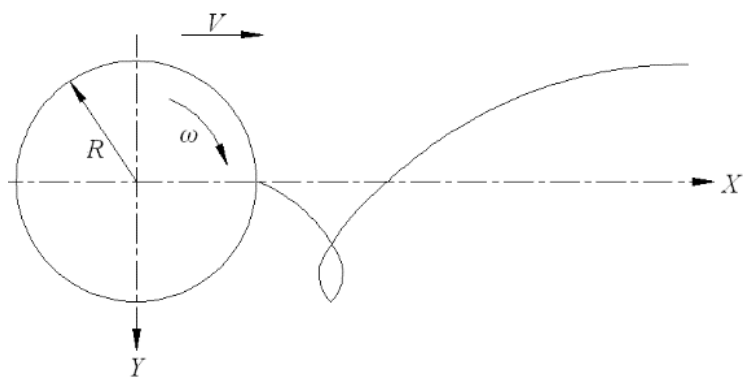

Figure 5.The locus curve of a point on cutting disc

While cutting residues, the disc moves at a constant speed $V=2.5 \mathrm{~m} / \mathrm{s}$ along the positive axis $\mathrm{x}$, meanwhile making pure rolling at the central axis, so the movement locus of any point at the disc is trochoid,as shown in Fig.5.

By ProE, locus curves of four points, labeled 1,2,3,4, at the disc with incidence angle of ripples $\theta=30^{\circ}$ were charted in Fig.6, at the same way, locus curves of four points, labeled $1^{\prime}, 2^{\prime}, 3^{\prime}, 4^{\prime}$ at the disc with $\theta=0^{\circ}$ was charted in Fig.7, that of four points, labeled $1^{\prime}, 2^{\prime}, 3^{\prime}, 4^{\prime}$ at the disc with $\theta=45^{\circ}$ was Fig. 8 .

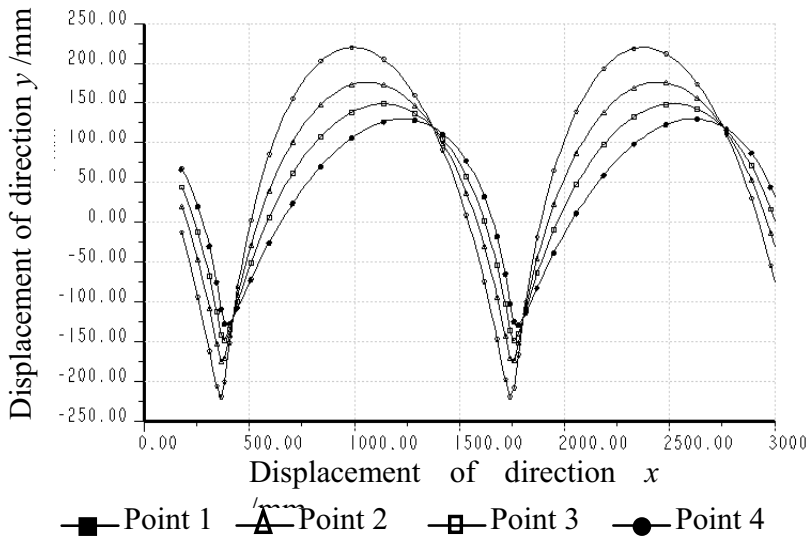

Figure 6.Movement locus of points on disc with $\theta=30^{\circ}$

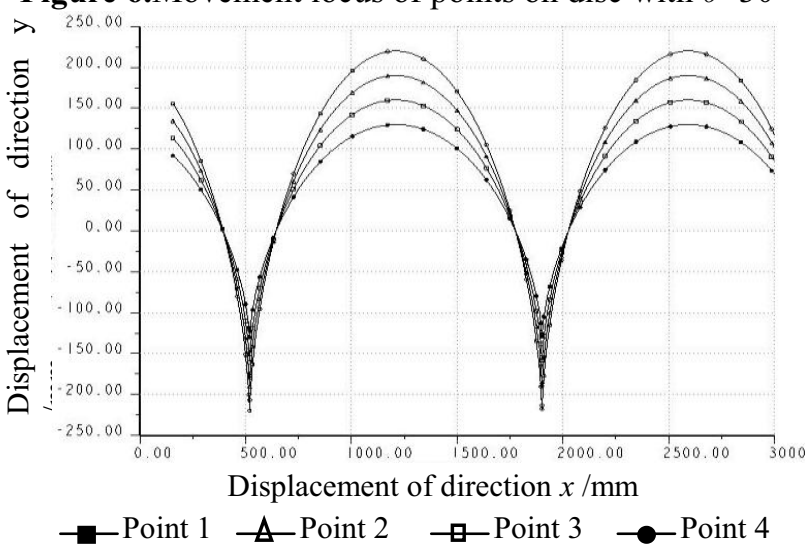

Figure 7.Movement locus of points on disc with $\theta=0^{\circ}$ 


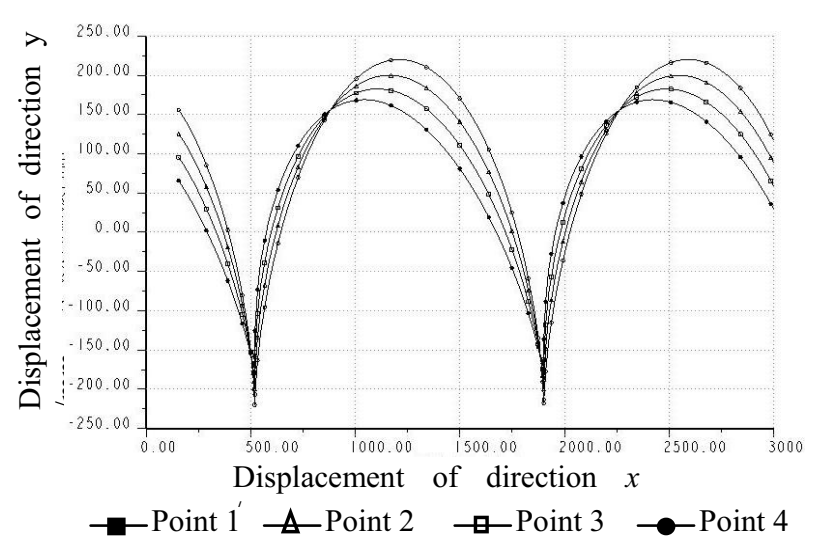

Figure 8.Movement locus of points on disc with $\theta=45^{\circ}$

\section{Stress analysis of disc}

\subsection{Force model}

While working as shown in Fig.9, the cutting-disc is acted upon by support force $F_{\mathrm{Z}}$, lateral compression $\mathrm{F}_{\mathrm{N}}$, and rotarycutting resistance $\mathrm{F}_{f}$. Support force $\mathrm{F}_{Z}$, in radial direction, is exerted by soil foundation to sustain no-tillage planter. Stubble and soil exert lateral compression $\mathrm{F}_{\mathrm{N}}$ perpendicular to both sides of the ripple surface. Rotary cutting resistance $\mathrm{F}_{f}$ distributes on the working ripple surface along peripheral direction.

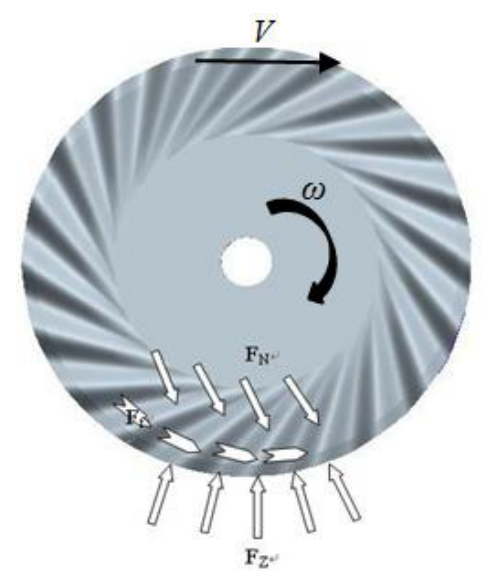

Figure 9.Force diagram of cutting disc

According to the detail design scheme of planter and actual working condition, the portion of the total planter weight assigned to a cutting-disc is about $160 \mathrm{~kg}$, so $F_{\mathrm{Z}}=1600 \mathrm{~N}$.

Lateral compression $F_{N}$ :

$$
F_{N}=P A(1)
$$

where, $P$-integrated deformation resistance of soil and stubble

$A$-the force bearing area

By field tests, the force bearing area is about $73.8 \mathrm{~cm}^{2}$, $A=73.8 \mathrm{~cm}^{2}$. Lateral compression can be obtained by formula(1), $F_{N}=1048 \mathrm{~N}$.

Rotary cutting resistance $\mathrm{F}_{f}$ consists of the friction and adhesive force, actually, $\mathrm{F}_{f}$ is calculated by the following empirical formula,

$$
F_{f}=\mu^{\prime \prime} F_{N}(2)
$$

where, $\mu^{\prime \prime} \_$practically syntheticalcoefficient

$F_{N}$ lateral compression

According to W.R $(1983)^{1}, \mu^{\prime \prime}=0.35 \sim 0.8$ for clay, here we choose $\mu^{\prime \prime}=0.7$.According to sticky soil in north China, integrated deformation resistance of soil and stubble is $P=1.42 \mathrm{~kg} / \mathrm{cm}^{2}$. According toformula(2), $\mathrm{F}_{f}=733.73 \mathrm{~N}$.

\subsection{Stress analysis based on ANSYS}

\subsubsection{Modelling and meshing}

The disc is modeled and meshed by ANSYS 10.1, as shown in Fig. 10. Use the following Settings:

Material:65Mn, elastic modulus EX:210GPa, Poisson's ratio NUXY:0.3.

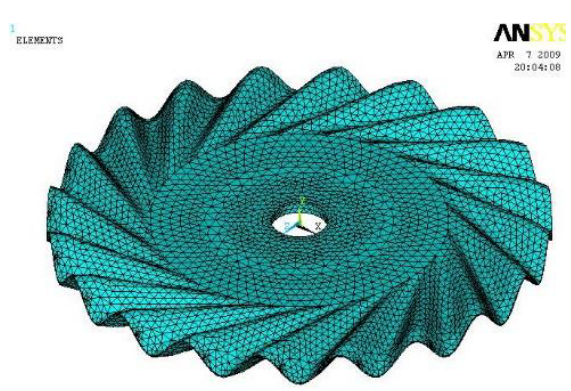

Figure 10.Modelling and meshing

\subsubsection{Loading}

Field testing shows that eight ripples are simultaneously in the state of cutting residues and soil,so based on value and direction of force model discussed in Section 3.1, support force $F_{\mathrm{Z}}$, lateral compression $\mathrm{F}_{\mathrm{N}}$ and rotary cutting resistance $\mathrm{F}_{f}$ are applied on the lower eight ripples, as shown in Fig.11.

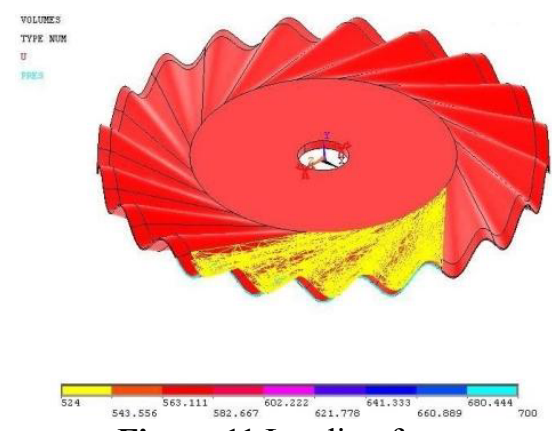

Figure 11.Loading force

\subsubsection{FEA results}

Stress intensity and shearing stress of $X Z$-direction are charted as followed.

1. See Fig.12, the flat part of disc is in the slightly stress condition, stress mainly distributes on the lower eight 
ripples, the largest stress, which is $3490 \mathrm{~Pa}$, is on the ripple which splits the soil.

2. As shown in Fig.13, the maximum shearing stress is both on the ripple which splits the soil, and on the cutting-edge near the ripple which splits the soil, but the maximum shearing stress is very small, only 535.83Pa.

3. Comprehensively, the weak part of the disc is the ripple, the flat part is in the very safe state.

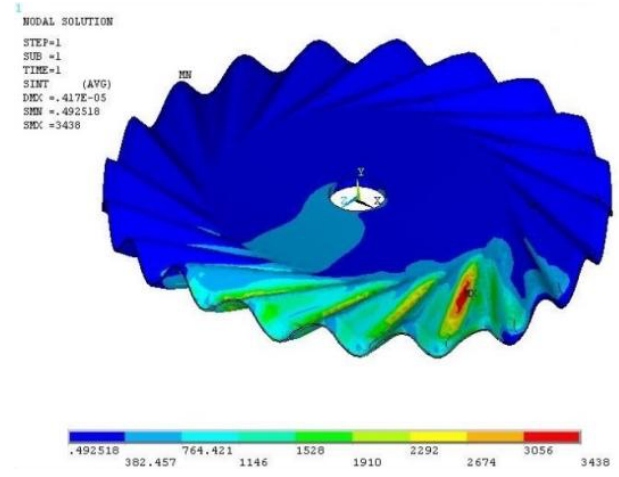

Figure 12.Stress intensity nephogram

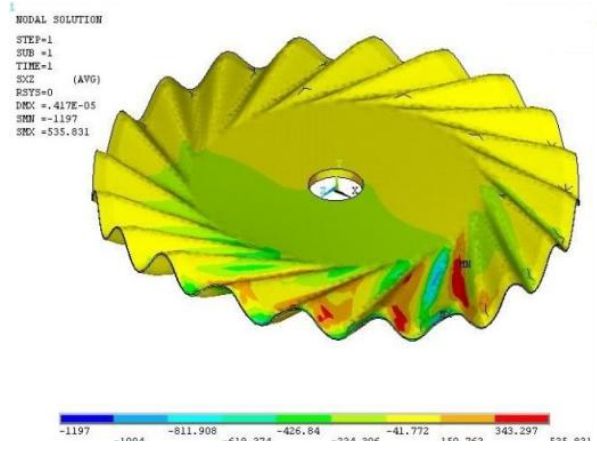

Figure 13.Shearing stress nephogram of $X Z$-direction

\subsubsection{Thermal treatment plan}

Based on the above analysis, the thermal treatment plan is as followed:

The whole: Salt bath quenching + medium-temperature tempering, the material microstructure is tempered troostiteafter heat treatment, the yield limit and impact toughness rise.

Ripples: After the thermal treatment, cold drawn is applied to strengthen toughness and plasticity. Moreover, by means of laser surface strengthening with $1200 \mathrm{~W}$ power at speed $20 \mathrm{~mm} / \mathrm{s}$, intensity and wear-resistance are enhanced.

\section{Conclusions}

Cutting disc with oblique ripples is a crucial, effective type of cutting-residues and anti-blocking. After the decision of disc's elementary structure excluding ripplesangle,kinematics analysis of discs with different rippleangle is conducted to determine the optimum rippleangle, the result showed that cutting property of disc cutter with $30^{\circ}$-obliquity ripples is best than others. And FEA(finite element analysis) shows that the largest stress is on the ripple which splits the soil, thermal treatment plan is drawn up based on the FEA, such as salt bath quenching and medium-temperature tempering for the whole, and cold drawn and laser surface strengthening is applied on the ripples to enhance the toughness and wear-resistance.

\section{References}

1. Wilkins DE, Muilenburg GA, et al,Transaction of the ASAE 26,651-660(1983)

2. Zhang Jinguo, GaoHuanwen, Proceeding of int. conf. agri. Eng. 99-ICAE, 326-332(1999)

3. LN Shukla, AM Chauhan, LS Dhaliwal, AMA 27,15-18(1999)

4. JP Gupta, KP Pandey, Agr. Mech. Asia, Africa and Latin America 27,16-20(1996)

5. JA Smith, RG Wilson, GD Binford, CD Yonts, Ame. Soc. Agric. Eng.18,667-672(2002)

6. McLeod, JG, Dyck FB, Campbell CA, Soil Till. Res 25,1-16 (1992)

7. T Vamerali, M Bertoccob, L Sartori, Soil \& Till. Res.89,196-209(2006)

8. Baker CJ, Saxton KE, Ritchie WR , Cab Int1937,245-252 (1996)

9. Lin Jing, Qian Wei, Li Baofa, Trans. Chin. Soc. Agric. Eng. 31,19-24(2015)

10. DavutKarayel, EgidijusSavaushis, Agric. Eng. , 43,16-24(2011)

11. Dun Guoqiang, Chen Haitao, Li Ang, Trans. Chin. Soc. Agric. Eng. 31,48-56(2015)

12. Lin Jing, Li Bo, Li Baofa, Trans. Chin. Soc. Agric. Mech., 45,118-124(2014)

13. Yang Zidong, Liu Ningning, GengDuanyang, Trans. Chin. Soc. Agric. Mech., 44,46-50(2013) 